\title{
Loop Quantum Cosmology: Recent Progress
}

\author{
Martin Bojowald \\ Max-Planck-Institut für Gravitationsphysik, \\ Albert-Einstein-Institut, 14476 Potsdam, Germany
}

\begin{abstract}
Aspects of the full theory of loop quantum gravity can be studied in a simpler context by reducing to symmetric models like cosmological ones. This leads to several applications where loop effects play a significant role when one is sensitive to the quantum regime. As a consequence, the structure of and the approach to classical singularities are very different from general relativity: The quantum theory is free of singularities, and there are new phenomenological scenarios for the evolution of the very early universe including inflation. We give an overview of the main effects, focussing on recent results obtained by several different groups.

PACS numbers: 04.60.Pp,98.80.Bp,98.80.Qc
\end{abstract}




\section{INTRODUCTION}

One of the leading contenders for a quantum theory of gravity is loop quantum gravity, which is a canonical quantization of general relativity formulated in Ashtekar's variables. With its properties of being background independent and non-perturbative it is well-equipped for the understanding of extreme physical situations such as black holes and the big bang. On the other hand, special techniques are required which are essential for a well-defined framework but often unfamiliar. These techniques and their implications can be illustrated and tested in simple situations by introducing symmetries, which is the origin of loop quantum cosmology. The symmetry reduction can be done in such a way that the characteristic basic features of the full theory survive in explicit models, which exhibit far-reaching consequences even with contact to observations.

This overview starts with a brief introduction to loop quantum gravity and its symmetric cosmological models. Early results of isotropic loop quantum cosmology were the observation that the evolution equation becomes discrete and non-singular, and that operators for inverse powers of the volume are bounded with a modified behavior at small scales (these aspects have been reviewed in more detail in [1]). The modified inverse volume is a nonperturbative effect of the quantum theory, but it can nevertheless be studied in effective classical systems which are easier to analyze. At the same time, the quantization is subject to ambiguities leading to several parameters in the resulting expressions which can be exploited in phenomenological investigations.

The methods have been extended to anisotropic models and lead in particular to a Bianchi IX dynamics which is not chaotic thanks to small-scale modifications. The effective classical equations give an intuitive illustration of the modified appeoach to the classical singularity. Also the isotropic behavior is different at small scales if matter is present, which can be illustrated in a bounce picture replacing the big crunch. The same modified dynamics also has consequences for inflationary scenarios with characteristic deviations from the standard case at early stages. This can leave observable imprints on the microwave background at large scales. Work in progress includes a systematic derivation of perturbative corrections as well as approaches to find the physical inner product and observables. 


\section{LOOP QUANTUM GRAVITY}

Loop quantum gravity [2, 3] is based on Ashtekar's variables [4, 5] which are a densitized triad $E_{i}^{a}$ and the $\mathrm{SU}(2)$-connection $A_{a}^{i}=\Gamma_{a}^{i}-\gamma K_{a}^{i}$ where $\Gamma_{a}^{i}$ is the spin connection compatible with the triad and $K_{a}^{i}$ the extrinsic curvature. The Barbero-Immirzi parameter $\gamma$ can take any positive real value which does not influence the classical theory. The main advantage of those variables for a canonical quantization is that gravity now appears in the form of a gauge theory with a compact gauge group (the group of triad rotations) for which there are powerful background independent quantization techniques. For Einstein's field equations additional constraints, the diffeomorphism and the Hamiltonian constraint, have to be imposed.

To obtain a mathematically well-defined framework one chooses appropriate basic objects which are constructed from the fields $A_{a}^{i}$ and $E_{i}^{a}$ on a space manifold $\Sigma$. The basic ingredient of a loop quantization [6, 7] is to use holonomies $h_{e}(A)=\mathcal{P} \exp \int_{e} A_{a}^{i} \dot{e}^{a} \tau_{i} d t \in \mathrm{SU}(2)$ and fluxes $F_{S}(E)=\int_{S} E_{i}^{a} n_{a} \tau^{i} d^{2} y$ where $\tau_{i}$ are Pauli matrices, $\dot{e}^{a}$ is the tangent vector to the edge $e$ and $n_{a}$ the co-normal to the surface $S$. If all curves $e$ and surfaces $\Sigma$ are allowed, holonomies and fluxes contain the same information as the original fields. For a quantization, however, holonomies and fluxes are much better since they are smeared versions of the fields obtained by natural integrations along curves and surfaces. In contrast to a threedimensional smearing, which is usually employed in quantum field theory, the one- and two-dimensional smearings can be done without introducing a background metric. This is essential for a background independent quantization.

The quantum theory is then defined on a representation of the holonomy-flux Poisson-

* algebra, which also needs to be background independent. It has recently been shown that such a representation is unique [8, 9, 10, 11]. States can most easily be given in the connection representation as gauge invariant functions of the connection via holonomies. A basis is given by spin network states [12] which are associated with graphs in $\Sigma$ whose edges are labeled by irreducible $\mathrm{SU}(2)$-representations. This indicates that such a state depends on the connection only via the holonomies along edges of the graph, each of which is taken in the corresponding representation. The matrices are then contracted to a gauge invariant function, which requires additional labels in vertices if their valence is larger than three. This state space carries a diffeomorphism invariant inner product, the Ashtekar- 
Lewandowski inner product [13]. Basic operators are matrix elements of holonomies which act by multiplication, and fluxes as derivative operators which can be seen to count the intersection number of the surface associated with the flux and the graph associated with the state being acted on.

There are three characteristic features of this representation, the hallmarks of any loop quantization:

1. The Hilbert space before imposing the constraints is non-separable, owing to the fact that all spin network states with different graphs, which form a continuous set of labels, are orthogonal to each other.

2. Holonomies are well-defined operators by definition, but it is not possible to represent the connection $A_{a}^{i}$ itself as an operator.

3. Flux operators have discrete spectra as the intersection numbers above. This entails a discrete spatial geometry [14, 15, 16] since the triad contains all information about the geometry of space.

So far, only the basic operators have been described which now have to be used to construct more complicated ones. In particular one has to represent and solve the diffeomorphism and Hamiltonian constraint to implement Einstein's equations. This can be done for the diffeomorphism constraint [7], and there are well-defined operators for the Hamiltonian constraint [17]. The latter, however, are extremely complicated and not much is known about their solution space. At this point it is advantageous to introduce symmetries in order to apply the theory in a simpler context.

\section{LOOP QUANTUM COSMOLOGY}

Classically, symmetries are introduced simply by restricting to symmetric basic fields. In the case of isotropy we have an isotropic triad $E_{i}^{a}=p \Lambda_{i}^{I} X_{I}^{a}$ and connection $A_{a}^{i}=c \Lambda_{I}^{i} \omega_{a}^{I}$ where $\omega^{I}$ and $X_{I}$ are left-invariant (under the action of the symmetry group) 1-forms and vector fields and $\Lambda$ is an $\mathrm{SO}(3)$-matrix indicating the internal su(2)-direction of the components. All these ingredients do not play a physical role; the former are given once we choose the symmetry group and the latter can be chosen freely and are pure gauge degrees 
of freedom. The only physical components are $p$ and $c$ which are related to the more familiar scale factor by the relations $|p|=a^{2}$ and $c=\frac{1}{2}(k-\gamma \dot{a})$ where $k$ can be zero for a flat model or one for a closed model.

On these variables we have to impose the Hamiltonian constraint

$$
12 \gamma^{-2}\left[c(c-k)+\left(1+\gamma^{2}\right) k^{2} / 4\right] \sqrt{|p|}=8 \pi G H_{\text {matter }}\left(p, \phi, p_{\phi}\right)
$$

where $G$ is the gravitational constant and $H_{\text {matter }}$ the matter Hamiltonian which depends on the metric and also on matter fields and their momenta simply denoted as $\phi$ and $p_{\phi}$. Using the relations to the scale factor one can see immediately that the constraint is nothing but the Friedmann equation

$$
3\left(\dot{a}^{2}+k^{2}\right) a=8 \pi G a^{3} \rho_{\text {matter }}\left(a, \phi, p_{\phi}\right) .
$$

Loop quantum cosmology is based on symmetric states in loop quantum gravity which by definition are supported only on invariant connections [18, 19]. Thus, all holonomies are of the form

$$
h_{I}=\exp \left(c \Lambda_{I}^{i} \tau_{i} \int \omega^{I}\right)=\cos (\mu c / 2)+2 \Lambda_{I}^{i} \tau_{i} \sin (\mu c / 2)
$$

where $\mu \in R$ is a real parameter for the edge length. Gauge invariant isotropic states can only depend on the connection via those holonomies such that they are of the form $\psi(c)=\sum_{\mu} \psi_{\mu} e^{i \mu c / 2}$ with a sum over a countable subset of the real line. With the notation $\langle c \mid \mu\rangle:=e^{i \mu c / 2}$ the inner product is $\left\langle\mu \mid \mu^{\prime}\right\rangle=\delta_{\mu \mu^{\prime}}$. Basic operators are the holonomies $e^{i \mu^{\prime} c / 2}|\mu\rangle=\left|\mu+\mu^{\prime}\right\rangle$ and the flux $\hat{p}|\mu\rangle=\frac{1}{6} \gamma \ell_{\mathrm{P}}^{2} \mu|\mu\rangle$.

This representation has the same properties as observed before for loop quantum gravity in general [20]: There is a non-separable Hilbert space, only holonomies but not $c$ itself are promoted to well-defined operators, and the flux $\hat{p}$ has a discrete spectrum. It is not possible to derive a $c$-operator because the holonomy operators are not continuous in $\mu$ at $\mu=0$ such that the derivative does not exist. The flux has a discrete spectrum in the sense that it has normalizable eigenstates $|\mu\rangle$, even though the range of eigenvalues is continuous, the real line.

Comparing to a Wheeler-DeWitt quantization, on the other hand, shows a completely different framework: the representations are inequivalent. Furthermore, a Wheeler-DeWitt quantization would have a well-defined operator for $c$ (which in this context is equivalent to the extrinsic curvature), and a continuous spectrum of $p$ or the scale factor $a$. Both these 
new features of the loop quantization have significant further consequences. (One can obtain a similar representation also with ADM variables, as done explicitly in [21].)

\section{A. Discrete evolution}

Since there is no $c$-operator, the constraint (11) has to be quantized in an indirect way using holonomies. The resulting quantized Friedmann equation becomes a difference equation which shows drastic differences to the Wheeler-DeWitt equation at small volume. As a consequence, cosmological singularities are absent, which has been shown for all homogeneous models [22, 23, 24, 25, 26]. As an example we discuss the isotropic equation

$$
\begin{aligned}
& \left(V_{\mu+5}-V_{\mu+3}\right) e^{i k} \psi_{\mu+4}(\phi)-\left(2+\gamma^{2} k^{2}\right)\left(V_{\mu+1}-V_{\mu-1}\right) \psi_{\mu}(\phi) \\
& +\left(V_{\mu-3}-V_{\mu-5}\right) e^{-i k} \psi_{\mu-4}(\phi)=-\frac{4}{3} \pi \gamma^{3} G \ell_{\mathrm{P}}^{2} \hat{H}_{\text {matter }}(\mu) \psi_{\mu}(\phi)
\end{aligned}
$$

for the wave function $\psi_{\mu}(\phi)$, where $V_{\mu}=\left(\gamma \ell_{\mathrm{P}}^{2}|\mu| / 6\right)^{3 / 2}$ are the volume eigenvalues and $\hat{H}_{\text {matter }}(\mu)$ the matter Hamiltonian acting on the wave function.

As a recurrence relation, this equation does not break down when we reach the classical singularity $\mu=0$, and it allows us to evolve from positive $\mu$ to negative $\mu$ reaching a branch preceding the classical singularity in internal time $\mu$ 22]. Essential for this property is the fact that the matter Hamiltonian satisfies $\hat{H}_{\text {matter }}(0)=0$ when it corresponds to to quantized matter and geometry, e.g. $\hat{H}_{\text {matter }}=\frac{1}{2} \widehat{a^{-3}} \otimes \hat{p}_{\phi}^{2}+\hat{a}^{3} \otimes W(\phi)$ for a scalar. This property will be shown in the next subsection.

\section{B. Finite inverse scale factor operator}

For the matter Hamiltonian we need a quantization of $a^{-3}$, for which we could try to use an inverse power of the flux $\hat{p}$. However, since the flux has a discrete spectrum containing zero, its inverse does not exist as a densely defined operator. There is an alternative procedure to quantize $a^{-3}$ which comes directly from the full theory [27]. One first rewrites the object in a way which is better suited for quantization, e.g. $a^{-3}=\left(3(8 \pi \gamma G l)^{-1}\left\{c,|p|^{l}\right\}\right)^{3 /(2-2 l)}$ where we do not need any inverse power if $0<l<1$ (a comparison with the full theory shows that some

values for $l$ appear more naturally, e.g. $l=\frac{3}{4}$ if one uses the formulas of $27 \mid$; the resulting expressions for this value have been used in recent applications [28, 29, 30]). Now the 
connection component appears for which we do not have an operator. This problem can again easily be avoided by using holonomies, $a^{-3}=\left((4 \pi \gamma G l)^{-1} \sum_{I} \operatorname{tr}\left(\Lambda_{I}^{i} \tau_{i} h_{I}\left\{h_{I}^{-1},|p|^{l}\right\}\right)\right)^{3 /(2-2 l)}$. This expression can now be quantized immediately by using holonomy and flux operators and turning the Poisson bracket into a commutator. The result is a well-defined, finite operator which does not show the classical divergence of $a^{-3}$ at $a=0$ [31]. However, since we had to reformulate the non-basic classical expression there is room for several quantization ambiguities. One of them is the parameter $l$ which, as we will see, has an effect on the quantization. Another ambiguity comes from taking the trace, which can be done in any $\mathrm{SU}(2)$-representation, not just the fundamental one as understood above.

If all these ambiguities are implemented, we obtain the eigenvalues

$$
\left(\widehat{a^{-3}}\right)_{\mu}^{(j, l)}=\left(\frac{9}{\gamma \ell_{\mathrm{P}}^{2} l j(j+1)(2 j+1)} \sum_{k=-j}^{j} k\left|p_{\mu+2 k}\right|^{l}\right)^{\frac{3}{2-2 l}}
$$

with $p_{\mu}=\frac{1}{6} \gamma \ell_{\mathrm{P}}^{2} \mu$. For larger $j$, the sum can be approximated well by 32 ]

$$
\left(a^{-3}\right)_{\mathrm{eff}}^{(j, l)}(a):=\left(\widehat{a^{-3}}\right)_{\mu\left(a^{2}\right)}^{(j, l)}=a^{-3} p_{l}\left(3 a^{2} / \gamma j \ell_{\mathrm{P}}^{2}\right)^{3 /(2-2 l)}
$$

with $\mu(p)=6 p / \gamma \ell_{\mathrm{P}}^{2}$ and

$$
\begin{aligned}
p_{l}(q)=\frac{3}{2 l} q^{1-l} & \left((l+2)^{-1}\left((q+1)^{l+2}-|q-1|^{l+2}\right)\right. \\
& \left.-(l+1)^{-1} q\left((q+1)^{l+1}-\operatorname{sgn}(q-1)|q-1|^{l+1}\right)\right) .
\end{aligned}
$$

The functions $p_{l}(q)$ approach one for $q \gg 1$ but are increasing with power $p_{l}(q) \sim 3(l+$ $1)^{-1} q^{2-l}$ for $q \ll 1$. The transition between the two regimes takes place around $q \approx 1$ (see Fig. 1). For the effective density $\left(a^{-3}\right)_{\text {eff }}^{(j, l)}$ this implies that it does not diverge at $a=0$ but rather approaches zero and is increasing as

$$
\left(a^{-3}\right)_{\mathrm{eff}}^{(j, l)} \sim a^{3 /(1-l)} \quad \text { for } a \ll \sqrt{\gamma j} \ell_{\mathrm{P}}
$$

resulting in a power law behavior with some power larger than three at small volume; see Fig. 1. (The power, however, is not constant but decreases with increasing volume such that stability issues like those in ordinary super-inflation are avoided.) Unlike the classical reformulation of $a^{-3}$, the formula for the eigenvalues can also be applied in the limit $l \rightarrow 0$, which results in

$$
p_{0}(q):=\lim _{l \rightarrow 0} p_{l}(q)=\frac{3}{2} q\left(q+\frac{1}{2}\left(1-q^{2}\right) \log \frac{q+1}{|q-1|}\right) .
$$



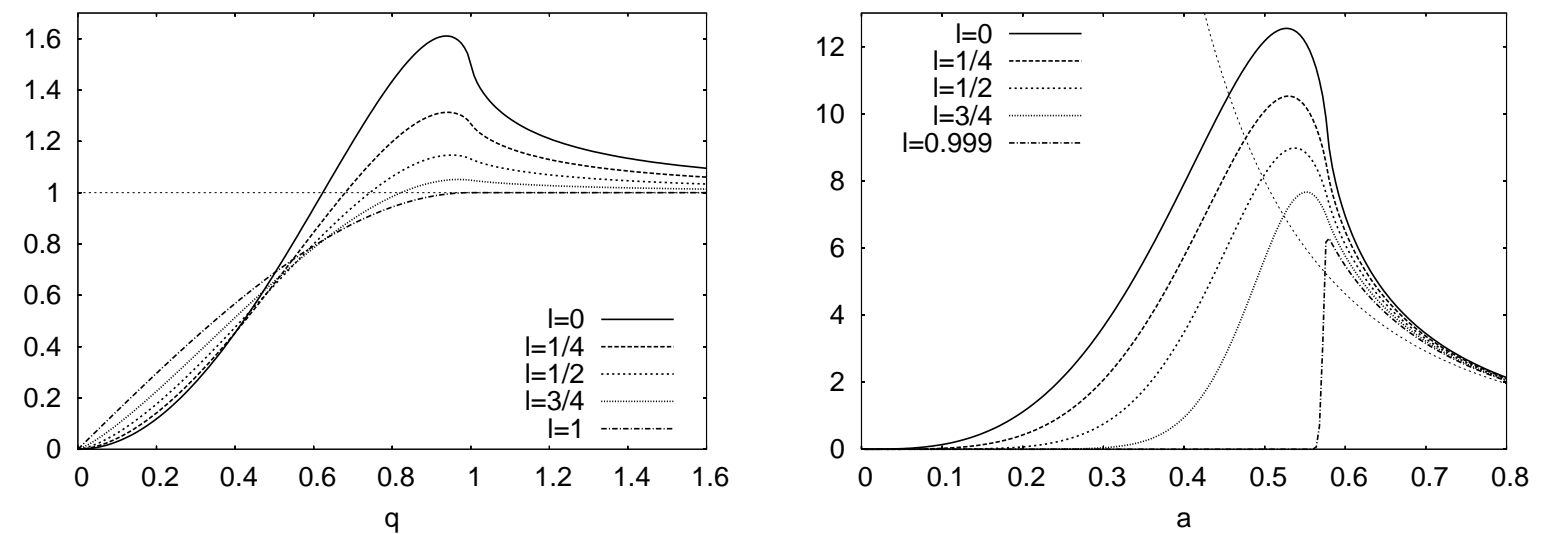

FIG. 1: The functions $p_{l}(q)$ and the corresponding effective densities in units of $\left(\sqrt{\gamma j} \ell_{\mathrm{P}}\right)^{-3}$, with $a$ in units of $\sqrt{\gamma j} \ell_{\mathrm{P}}$. Also shown are the classical expectations one for $p_{l}(q)$ and $a^{-3}$ for the density (dotted).

In this limiting case, the effective density behaves just like the volume at small scales.

The basic properties of the quantized $a^{-3}$ can be seen directly from these expressions:

$\mu=0$ : We have $\left(\widehat{a^{-3}}\right)_{\mu=0}^{(j, l)}=0$ since in this case we have a summation over an odd function in (15). This proves the claim made before that $\hat{H}_{\text {matter }}(0)=0$ since the metric operators in both the kinetic and the potential term vanish.

$0 \leq \mu \leq 2 j:\left(a^{-3}\right)_{\mathrm{eff}}^{(j, l)}(a)$ increases with $a$, which implies a modified Friedmann dynamics. In particular, the energy density increases with $a$ which implies inflation [28].

$\mu \approx 2 j:\left(a^{-3}\right)_{\mathrm{eff}}^{(j, l)}(a)$ has a peak where the transition to the classical $a^{-3}$ takes place.

This behavior is certainly very different from the one expected classically, but it is very characteristic for the loop effects. In fact, even though there are ambiguities like those indicated by the parameters $(j, l)$, the features discussed here are robust. There are more intuitive explanations for the effects which build on the experience with quantum mechanics: The peak implies a finite upper bound for $a^{-3}$ of the order $(\gamma j)^{-3 / 2} \ell_{\mathrm{P}}^{-3}$ which diverges in the classical limit $\ell_{\mathrm{P}} \rightarrow 0$, just as the finite ground state energy of the hydrogen atom, $E_{0}=-\frac{1}{2} m e^{4} / \hbar^{2}$ diverges in the classical limit.

Furthermore, the behavior around the peak provides an interpolation between inflationary behavior at small scales (energy increasing with $a$ ) and ordinary matter behavior at large 
volume which would otherwise diverge when followed through to the classical singularity. Thus, the energy density of matter in a universe behaves differently when quantum effects are taken into account. A similar situation is well-known for the energy density of radiation in a cavity. In fact, it is analogous to the black body radiation whose spectral energy density for large wave length, $\lambda \gg h / k T$, is well described by the Rayleigh-Jeans law $\rho_{T}(\lambda) \sim 8 \pi k T / \lambda^{2}$ with an unphysical divergence at $\lambda=0$. Quantum mechanics shows that the correct formula is given by Planck's expression, $\rho_{T}(\lambda)=8 \pi h \lambda^{-3}\left(e^{h / k T \lambda}-1\right)^{-1}=h \lambda^{-3} f\left(\lambda / \lambda_{\max }\right)$ with $\lambda_{\max }=$ $h / x k T, e^{x}(3-x)=3$, and $f(y)=8 \pi /\left((3 /(3-x))^{1 / y}-1\right)$. The peak position indicating the transition region is determined by the inverse temperature $T^{-1}$.

Formally, the effective density in loop quantum cosmology behaves similarly. The classical expression $a^{-3}$ is valid only at large $a$, while the full expression is given by (6) $),\left(a^{-3}\right)_{\text {eff }}^{(j, l)}(a)=$ $a^{-3} p_{l}\left(a^{2} / a_{\max }^{2}\right)^{3 /(2-2 l)}$, with a peak at $a_{\max }=\sqrt{\gamma j / 3} \ell_{\mathrm{P}}$. One could think that there is more than just a formal analogy and that $j$, which determines the peak, is related to an inverse temperature. In fact, if we choose the limiting case $l=0$, we have $\left(a^{-3}\right)_{\text {eff }}^{(j, 0)} \sim \Lambda_{\text {eff }} a^{3}$ with $\Lambda_{\text {eff }} \propto a_{\max }^{-6}$. In this case, the limiting behavior is asymptotically de Sitter at small $a$, which is known to have a temperature $T=\sqrt{\Lambda} / 2 \sqrt{3} \pi$ [33]. With $\Lambda_{\text {eff }}$, this becomes $T \propto a_{\max }^{-3} \propto j^{-3 / 2} \ell_{\mathrm{P}}^{-3}$ such that for the volume we have $V_{\max } / V \propto 1 / V T$ and we can write the effective density as $\left(a^{-3}\right)_{\text {eff }}^{(j, 0)}(V)=V^{-1} g(V T)$ with a suitable function $g$, just as in the case of the black body spectrum with $\lambda$ replacing $V$. At this point, however, there is no indication that the ambiguity parameter $j$ can play such a physical role analogous to the temperature.

\section{RECENT APPLICATIONS}

Recently the original results of loop quantum cosmology have been extended and studied in more detail, and more work is in progress. The current research can mainly be grouped into the following categories.

\section{A. Quantum structure of classical singularities}

For all homogeneous models that can be treated canonically (Bianchi class A models) the absence of cosmological singularities can be shown along the same general scheme as in 
the isotropic case [25, 26]. The quantum dynamics is given by a difference equation which does not break down at the classical singularity but instead extends to a branch beyond the classical singularity. The new branch is always provided by triads of reversed orientation, just as the sign of $\mu$ indicates the orientation in the isotropic case.

To get hints as for the behavior of general, inhomogeneous singularities, one can make use of the BKL scenario [34]. One then views space approximately as being composed of almost homogeneous patches each of which follows the most general homogeneous behavior: the Bianchi IX model. Since this model is chaotic, patches with initially similar geometries will depart rapidly from each other such that all the patches will need to be subdivided to maintain the approximation. Thus, the closer one comes to the classical singularity the more fragmented space becomes without bound, which is not only complicated but would also be inconsistent with a discrete structure such as that of loop quantum gravity.

In this argument, however, only classical properties of the model have been used. In particular the chaos comes from unbounded, diverging curvature which leads to reflections in a complicated potential with moving walls. From loop effects the curvature, just as the effective density before, will be bounded and the walls will even disappear at some finite volume [35]. The complicated chaotic behavior then stops at the discrete scale at the latest, making the loop scenario consistent.

This argument is based on effective classical equations of motion which have been modified by including the bounded curvature expressions. Such a procedure is valid at reasonably large volume where the classical trajectories describe the position of quantum wave packets. Similarly, one can develop a classical picture of the isotropic bounce. The classical equations are then modified by using the effective matter Hamiltonian $\frac{1}{2}\left(a^{-3}\right)_{\text {eff }} p_{\phi}^{2}+a^{3} W(\phi)$ using the expression (6) . This leads directly to the effective Friedmann equation

$$
3\left(\dot{a}^{2}+k^{2}\right) / a^{2}=8 \pi G\left(\left(a^{3}\left(a^{-3}\right)_{\mathrm{eff}}\right)^{-1} \dot{\phi}^{2} / 2+W(\phi)\right)
$$

and, via Hamiltonian equations of motion, to the modified Klein-Gordon equation [24]

$$
\ddot{\phi}=\dot{\phi} \dot{a} \frac{d \log \left(a^{-3}\right)_{\mathrm{eff}}}{d a}-a^{3}\left(a^{-3}\right)_{\mathrm{eff}} W^{\prime}(\phi)
$$

The main effect in the Klein-Gordon equation is that the friction term can now change its sign in the transition region of $\left(a^{-3}\right)_{\text {eff }}$ such that friction and antifriction for the scalar will be interchanged. When we evolve toward a classical big crunch, we have $\dot{a}<0$ such that 
classically with $d \log \left(a^{-3}\right) / d a=-3 / a<0$ we have antifriction. When we reach the classical singularity, $\phi$ diverges as a consequence. With the effective quantum behavior, however, $d \log \left(a^{-3}\right)_{\text {eff }} / d a$ will become positive once we reach small $a$. At this point, antifriction turns into friction which leads to $\phi$ being frozen at a finite value. This, in turn, implies that $\dot{\phi} \approx 0$ such that only the potential term of the matter Hamiltonian is relevant and the Friedmann equation gives a de Sitter bounce at non-zero $a$. This scenario has been observed and studied for different potentials in [30].

After the bounce, we have $\dot{a}>0$ and classically friction for the scalar, which is used in the slow-roll regime of inflation. With the effective density, at small scales we have antifriction instead such that $\phi$ will be driven away from the minima of its potential. This has further consequences for cosmological phenomenology.

\section{B. Phenomenology}

We already mentioned that the increasing behavior of $\left(a^{-3}\right)_{\text {eff }}$ at small volume implies, via the kinetic term of the matter Hamiltonian, inflation [28]. There is no graceful exit problem since inflation ends automatically once the scale factor reaches the peak value of the effective density where it starts its classical decrease. However, the usual cosmological perturbation theory is unstable in this regime 29] such that at this point we cannot tell if this inflationary phase is viable for structure formation. This phase will be present for any matter content, even without an inflaton field. If we do couple an inflaton with a potential suitable for chaotic inflation, there will be the second effect of the inflaton being driven up its potential by the antifriction term in its Klein-Gordon equation. Thus, starting from its initial values, $\phi$ increases early before at later times it reaches its maximal value which can be very large. This can provide the initial conditions necessary for chaotic inflation [28, 29]. After reaching the maximum, $\phi$ turns around and enters the slow-roll phase of standard inflation.

For observational purposes it is important to notice that around the turning point, where

$\dot{\phi} \approx 0$, the slow-roll conditions are violated [29]. Since this happens at the earliest stages of the slow-roll phase, it can lead to observable imprints such as a suppression of power and a running spectral index at large scales if the number of $e$-foldings is not too large. 


\section{Perturbative corrections}

So far, most applications have been concerned with the modified effective density since this is the most prominent effect. It is non-perturbative and can extend even to large volume if the parameter $j$ is chosen to be larger than one. Closer to the Planck scale there are also perturbative corrections from the discrete structure of the evolution equation [36]. They can be computed systematically from the expectation value of the Hamiltonian constraint operator in a coherent state peaked at a phase space point $\left(c_{0}, p_{0}\right)$. To leading order, the constraint equation then reduces to the Friedmann equation, but there are correction terms of two different kinds. In the flat isotropic model we have an asymptotic expansion of the form [37]

$$
\langle\hat{H}\rangle=-12 \gamma^{-2}\left(c_{0}^{2}\left(1+O\left(c_{0}^{2}\right)\right)+O\left(\ell_{\mathrm{P}}^{2} / p_{0}\right)+O\left(d^{-2}\right)\right) \sqrt{\left|p_{0}\right|}
$$

where the parameter $d$ characterizes the squeezing of the coherent state. The first two correction terms are familiar and can be interpreted as higher curvature corrections analogous to an effective action. The last term is unexpected from this point of view; its origin is the fact that there is no unique coherent state such as the Minkowski vacuum for an effective action. The squeezing parameter $d$ can be constrained by further physical considerations for the uncertainties of other quantities.

One may be tempted to relate this effective Hamiltonian to the isotropic part of an effective action with higher curvature corrections. In particular, local Lorentz invariance seems to be an issue since there is no guarantee that the corrections with powers of $c_{0}$, essentially the extrinsic curvature, will combine to the correct combinations of the four-dimensional curvature tensor. However, the relation between a perturbative effective Hamiltonian and a perturbative effective action is more subtle since higher curvature terms in the action imply higher derivatives. For higher derivatives, in turn, the Legendre transform of the perturbative action leads to a Hamiltonian which is not analytic in the expansion parameter [38]. From the canonical point of view, however, only the analytic part of the Hamiltonian can be seen in this perturbative analysis. Since only some terms of the curvature invariants contain higher derivatives, they will be broken apart by this procedure which can lead to apparent Lorentz violating terms in the effective Hamiltonian. Note also that doing a perturbation expansion does not commute with the Legendre transform for higher derivative theories such that a derivation of an effective action from the effective Hamiltonian is not straightforward. 


\section{Conceptual issues}

One of the most important open issues conceptually is that of the physical inner product and quantum observables. There are currently several stategies which are being followed in cosmological models, such as a Dirac analysis (see [39] for a preliminary investigation) and spin foam ideas.

This issue is also important in order to understand the number of independent solutions. Even in the isotropic vacuum case, the difference equation (4) has infinitely many solutions, all but two of which are rapidly oscillating at small scales and do not have a continuum approximation. The remaining two correspond to the two independent solutions of the Wheeler-DeWitt equation. They are further restricted by the quantum constraint resulting in only one linear combination [40,41]. The meaning of the infinitely many surplus solutions, however, and the possible role of superselection can only be understood with the help of observables and the physical inner product.

\section{CONCLUSIONS}

Loop quantum gravity takes the lesson of general relativity that the metric is dynamical and should be quantized without artificial background structures seriously. This requirement leads to a very rigid structure and can only be realized with special mathematical concepts. Chief among the basic mathematical properties are the fact that only holonomies will become well-defined operators rather than the connection or extrinsic curvature directly, and that the spatial geometry via fluxes acquires discrete spectra. Both these properties are also realized in loop quantum cosmology which allows reliable tests of the full theory in this simpler context, and also an investigation of further physical applications. As it turns out, the consequences of these two basic aspects, and the most obvious differences to a WheelerDeWitt quantization, are far-reaching.

The two properties entail immediately that the constraint (like other composite operators) has to be quantized using holonomies, and that the flux does not have an inverse operator such that its classical inverse has to be quantized after using a reformulation. Physically, this

implies that the quantum evolution equation is a difference rather than differential equation and that inverse powers of the scale factor are modified at small scales, removing the classical 
divergence. Both these effects are essential to show that the quantum evolution is nonsingular in all homogeneous models. On the other hand, they also lead to corrections to the classical equations of motion which are being used for phenomenological investigations. The difference equation leads to perturbative corrections which are relevant close to the Planck scale and so far have not been studied in detail. The modification of inverse powers of the scale factor, on the other hand, is non-perturbative and can extend into the semiclassical regime. Consequences reach from new solutions for conceptual problems to effects verging on observability.

It should be noted that all the modifications of the classical equations of motion are not chosen with a particular cosmological application in mind but rather derived from what we have learned about crucial properties of a background independent, non-perturbative quantization of general relativity. In all cases, the mathematical properties were known to be essential before they had been seen to lead to physical applications. In addition, there are now several independent properties which need to play together in a special way in order for the applications to hold true in the way described here. Comparing with the WheelerDeWitt quantization, furthermore, loop quantum cosmology has shown that inequivalent quantum representations of the same classical theory can have essentially identical behavior at large scales and at the same time lead to drastically different conclusions when small-scale properties are important.

\section{Acknowledgements}

The author is grateful to B. Iyer and the organizers of the International Conference on Gravitation and Cosmology (ICGC 2004), Cochin, India, for an invitation to a plenary talk and for providing a stimulating atmosphere for further discussions.

[1] M. Bojowald and H. A. Morales-Técotl, Cosmological applications of loop quantum gravity, In Proceedings of the Fifth Mexican School (DGFM): The Early Universe and Observational Cosmology, gr-qc/0306008

[2] C. Rovelli, Loop Quantum Gravity, Living Reviews in Relativity 1, 1 (1998), http://www.livingreviews.org/Articles/Volume1/1998-1rovelli, gr-qc/9710008 
[3] T. Thiemann, Introduction to Modern Canonical Quantum General Relativity, gr-qc/0110034

[4] A. Ashtekar, New Hamiltonian Formulation of General Relativity, Phys. Rev. D 36 (1987) $1587-1602$

[5] J. F. Barbero G., Real Ashtekar Variables for Lorentzian Signature Space-Times, Phys. Rev. D 51 (1995) 5507-5510, gr-qc/9410014

[6] C. Rovelli and L. Smolin, Loop Space Representation of Quantum General Relativity, Nucl. Phys. B 331 (1990) 80-152

[7] A. Ashtekar, J. Lewandowski, D. Marolf, J. Mourão, and T. Thiemann, Quantization of Diffeomorphism Invariant Theories of Connections with Local Degrees of Freedom, J. Math. Phys. 36 (1995) 6456-6493, gr-qc/9504018

[8] H. Sahlmann, Some Comments on the Representation Theory of the Algebra Underlying Loop Quantum Gravity, gr-qc/0207111

[9] H. Sahlmann, When Do Measures on the Space of Connections Support the Triad Operators of Loop Quantum Gravity?, gr-qc/0207112

[10] A. Okolow and J. Lewandowski, Diffeomorphism covariant representations of the holonomyflux star-algebra, Class. Quantum Grav. 20 (2003) 3543-3568, gr-qc/0302059

[11] H. Sahlmann and T. Thiemann, On the superselection theory of the Weyl algebra for diffeomorphism invariant quantum gauge theories, gr-qc/0302090

[12] C. Rovelli and L. Smolin, Spin Networks and Quantum Gravity, Phys. Rev. D 52 (1995) $5743-5759$

[13] A. Ashtekar and J. Lewandowski, Differential Geometry on the Space of Connections via Graphs and Projective Limits, J. Geom. Phys. 17 (1995) 191-230, hep-th/9412073

[14] C. Rovelli and L. Smolin, Discreteness of Area and Volume in Quantum Gravity, Nucl. Phys. B 442 (1995) 593-619, gr-qc/9411005, Erratum: Nucl. Phys. B 456 (1995) 753

[15] A. Ashtekar and J. Lewandowski, Quantum Theory of Geometry I: Area Operators, Class. Quantum Grav. 14 (1997) A55-A82, gr-qc/9602046

[16] A. Ashtekar and J. Lewandowski, Quantum Theory of Geometry II: Volume Operators, Adv. Theor. Math. Phys. 1 (1997) 388-429, gr-qc/9711031

[17] T. Thiemann, Quantum Spin Dynamics (QSD), Class. Quantum Grav. 15 (1998) 839-873, gr-qc/9606089

[18] M. Bojowald and H. A. Kastrup, Symmetry Reduction for Quantized Diffeomorphism Invari- 
ant Theories of Connections, Class. Quantum Grav. 17 (2000) 3009-3043, hep-th/9907042

[19] M. Bojowald, Quantum Geometry and Symmetry, Shaker-Verlag, Aachen, 2000

[20] A. Ashtekar, M. Bojowald, and J. Lewandowski, Mathematical structure of loop quantum cosmology, Adv. Theor. Math. Phys. 7 (2003) 233-268, gr-qc/0304074

[21] V. Husain and O. Winkler, On singularity resolution in quantum gravity, gr-qc/0312094

[22] M. Bojowald, Absence of a Singularity in Loop Quantum Cosmology, Phys. Rev. Lett. 86 (2001) 5227-5230, gr-qc/0102069

[23] M. Bojowald, Isotropic Loop Quantum Cosmology, Class. Quantum Grav. 19 (2002) 27172741, gr-qc/0202077

[24] M. Bojowald and K. Vandersloot, Loop quantum cosmology, boundary proposals, and inflation, Phys. Rev. D 67 (2003) 124023, gr-qc/0303072

[25] M. Bojowald, Homogeneous loop quantum cosmology, Class. Quantum Grav. 20 (2003) 25952615, gr-qc/0303073

[26] M. Bojowald, G. Date, and K. Vandersloot, Homogeneous loop quantum cosmology: The role of the spin connection, Class. Quantum Grav. 21 (2004) 1253-1278, gr-qc/0311004

[27] T. Thiemann, QSD V: Quantum Gravity as the Natural Regulator of Matter Quantum Field Theories, Class. Quantum Grav. 15 (1998) 1281-1314, gr-qc/9705019

[28] M. Bojowald, Inflation from quantum geometry, Phys. Rev. Lett. 89 (2002) 261301, gr-qc/0206054

[29] S. Tsujikawa, P. Singh, and R. Maartens, Loop quantum gravity effects on inflation and the CMB, astro-ph/0311015

[30] P. Singh and A. Toporensky, Big Crunch Avoidance in k = 1 Loop Quantum Cosmology, gr-qc/0312110

[31] M. Bojowald, Inverse Scale Factor in Isotropic Quantum Geometry, Phys. Rev. D 64 (2001) 084018, gr-qc/0105067

[32] M. Bojowald, Quantization ambiguities in isotropic quantum geometry, Class. Quantum Grav. 19 (2002) 5113-5130, gr-qc/0206053

[33] G. W. Gibbons and S. W. Hawking, Cosmological event horizons, thermodynamics, and particle creation, Phys. Rev. D 15 (1977) 2738-2751

[34] V. A. Belinskii, I. M. Khalatnikov, and E. M. Lifschitz, A general solution of the Einstein equations with a time singularity, Adv. Phys. 13 (1982) 639-667 
[35] M. Bojowald and G. Date, Quantum suppression of the generic chaotic behavior close to cosmological singularities, Phys. Rev. Lett. (2004) to appear, gr-qc/0311003

[36] M. Bojowald, The Semiclassical Limit of Loop Quantum Cosmology, Class. Quantum Grav. 18 (2001) L109-L116, gr-qc/0105113

[37] A. Ashtekar, M. Bojowald, and J. Willis, in preparation

[38] J. Z. Simon, Higher-derivative Lagrangians, nonlocality, problems, and solutions, Phys. Rev. D 41 (1990) 3720-3733

[39] G. M. Hossain, Hubble operator in isotropic loop quantum cosmology, Class. Quantum Grav. 21 (2004) 179-196, gr-qc/0308014

[40] M. Bojowald, Dynamical Initial Conditions in Quantum Cosmology, Phys. Rev. Lett. 87 (2001) 121301, gr-qc/0104072

[41] M. Bojowald, Initial Conditions for a Universe, Gen. Rel. Grav. 35 (2003) 1877-1883, gr-qc/0305069 